\title{
Influence of water-soluble nonionic polymers adsorption on colloidal properties of nanosilica dispersions
}

\author{
Olena V. Goncharuk ${ }^{\mathrm{a}, \mathrm{b}^{*}}$, Maria L. Malysheva ${ }^{\mathrm{c}}$, Konrad Terpilowski ${ }^{\mathrm{d}}$, \\ Salvador Pérez Huertas ${ }^{\mathrm{d}}$, Volodymyr M. Gun'ko ${ }^{\mathrm{a}}$ \\ ${ }^{a}$ Chuiko Institute of Surface Chemistry of NAS of Ukraine, Kyiv, Ukraine \\ ${ }^{\mathrm{b}}$ Ovcharenko Institute of Biocolloidal Chemistry of NAS of Ukraine, Kyiv, Ukraine \\ ${ }^{c}$ Department of Chemistry, Taras Shevchenko National University of Kyiv, Ukraine \\ ${ }^{\mathrm{d}}$ Faculty of Chemistry, Maria Curie-Sklodowska University, Lublin, Poland \\ iscgoncharuk@meta.ua
}

Keywords: polymer adsorption, nanosilica aqueous dispersion, poly(vinyl alcohol), poly(ethylene oxide), poly(vinyl pyrrolidone), aggregative and sedimentation stability, rheological properties.

The relationships between the adsorption of poly(vinyl alcohol) (PVA), poly(ethylene oxide) (PEO), and poly(vinyl pyrrolidone) (PVP) of various molecular weights onto nanosilica and the stability and rheological properties of the aqueous dispersions were analyzed. The adsorption isotherms for the polymers correspond to the Langmuir-type isotherms. The adsorption maximum slightly increases with increasing molecular weight of the polymers. The sedimentation and aggregative stability of the silica dispersion decreased at a low amount of an adsorbed polymer (less than a monolayer). At this polymer content, a significant increase in the viscosity of dispersions is observed due to the formation of polymeric bridges between silica nanoparticles from neighboring aggregates of them. If the amount of adsorbed polymer exceeds the monolayer then the stabilizing effect is observed due to the steric factor preventing the bridge formation and the viscosity of dispersion decreases slightly compared with systems with a low polymer content.

\section{Introduction}

The behavior of polymers at the interfaces of polymer solution/solid surface plays an important role in the properties of dispersions in liquid media and in the formation of filled polymer materials [1-7]. The adsorption of polymers leads to changes in the interactions of covered solid particles with neighboring particles and dispersion medium. These changes depend on the component types and concentrations, as well temperature, $\mathrm{pH}$ and salinity of the media.

Stabilization of aqueous dispersions by polymers is widely used in pharmacy for preparation of stable pharmaceutical substances, food industry, manufacture of products with a 
colloidal structure, etc. [1-5]. The opposite process of flocculation in similar systems is important too. It is used for purification of liquids from solid particles and other processes [6-9]. The mechanism of stabilization or flocculation of the colloidal particles by polymers is very complex and depends on many factors including the nature and concentration of polymers and concentration of solids [10-13]. Depending on the ratio of the parameters (it is unclear what means ratio of parameters), the disperse systems can be either stabilized or flocculated by polymers. Therefore, a study of the influence of the nature and concentration of the polymers on the stability and structural and mechanical properties of the dispersions is an important task from a practical point of view. Additionally, understanding of the structure formation processes occurring in complex colloidal systems in the presence of various polymers allow one a deeper insight into the phenomena.

The stabilizing or flocculating effects of polymers in colloidal systems is directly linked to the polymer adsorption onto the particles and structural features of formed adsorption layers. From a thermodynamic point of view, stronger interactions of polymer segments with active surface sites of solid particles than with neighboring molecules are the main driving forces of the polymer adsorption. A high molecular weight of macromolecules and flexibility of the polymer chains forming the loops and trains lead to only partial bonding of polymer functionalities to an adsorbent surface. Conformational constraints imposed by a surface of aggregated nanoparticles (as well narrow voids between nanoparticles in aggregates) and various nonlinear conformations of macromolecular chains in the solution result in the bonding only a relatively small part of the segments. This structural feature of polymer/nanoparticulate system is appropriate for the formation of polymeric bridges between solid nanoparticles [14]. On the other hand, it is possible steric stabilization of the system due to the penetration of nanoparticles into bulk polymer [1-5]. Therefore, the aim of this work was to study the effects of three types of non-ionic polar polymers adsorbed onto nanosilica on the properties of the aqueous systems (the aim of the work does not stem from the very general introduction).

\section{Experimental part}

\section{Materials and methods}

Fumed silica A-300 (pilot plant of the Chuiko Institute of Surface Chemistry, Kalush, Ukraine, specific surface area $\mathrm{S}=250 \mathrm{~m}^{2} / \mathrm{g}$ ) was used as the initial powder material.

Aqueous dispersions of nanosilica were prepared for rheology and stability investigations using distilled water. Silica concentration was a constant (5 wt. \%). After addition of silica to distilled water, the dispersions were sonicated for $3 \mathrm{~min}$ using an 
ultrasonic disperser UZDN-A (SELMI, Ukraine, 50-70 W, frequency $22 \mathrm{kHz}$ ). Then an amount of a polymer solution (concentration 5 wt. \%) was added to the dispersion to prepare the systems at various ratios of polymer and nanosilica. The rheological measurements were performed next day (to achieve the equilibrium state) after preparation of the dispersions.

Poly(ethylene oxide) (PEO) at molecular weight $\left(M_{\mathrm{w}}\right) 6,12$, and $100 \mathrm{kDa}$, poly(vinyl alcohol) (PVA) at $M_{\mathrm{w}}=43,60$, and $72 \mathrm{kDa}$, and poly(vinyl pyrrolidone) (PVP) at $M_{\mathrm{w}}=12,25$, and $55 \mathrm{kDa}$ were selected as water-soluble nonionic polar polymers.

\subsection{Adsorption studies}

Adsorption studies were carried out under equilibrium conditions at room temperature. A certain amount of the polymer solution (5 wt. $\%$ ) was added to $0.1 \mathrm{wt}$. \% dispersion of silica and mixed by a magnetic stirrer. The systems were equilibrated for one day. Then the silica dispersions were centrifuged, and the concentration of the polymer in the solution was determined using an interferometer. The value of the polymer adsorption onto a silica surface was calculated from the difference in the polymer concentrations in the solutions before and after contact with the adsorbent.

Changes in the Gibbs free energy $(\Delta G)$ upon polymer adsorption onto a solid surface from a solution can be calculated from the adsorption isotherm using the Langmuir equation

$$
\Theta=\frac{b C}{1+b C},
$$

where $b=\gamma_{L} e^{-\frac{\Delta G}{R_{g} T}}$ and $\gamma_{L}$ is a constant, using the minimization of the discrepancy functional for the experimental data and theoretical curve.

\subsection{Rheological measurements}

The rheological properties of the dispersions were studied using a rotational viscometer Rheotest 2.1 (cylinder system $\mathrm{S} / \mathrm{N}$ ) at shear rates from 9 to $1312.2 \mathrm{~s}^{-1}$. To determine the thixotropic properties of the dispersions, the rheological measurements were performed with stepwise increasing shear rate and exposure at maximal shear rate $\left(1312 \mathrm{~s}^{-1}\right)$ up to a constant viscosity, and finally stepwise decreasing shear rate. The results are shown as $2 \mathrm{D}$ and $3 \mathrm{D}$-graphs of the effective viscosity vs. the shear rate and time. This allows us to describe the destruction and restoration of the dispersion structure during the measurements. To compare different dispersions, the viscosity at the maximal shear rate (i.e. effective viscosity of the dispersion with most destroyed structure) was selected.

2.3. Aggregative and sedimentation stability

The stability study was carried out using a TurbiscanLabExpert with a TLab Cooler device. This apparatus possesses an electroluminescence diode which emits collimated light beam $(\lambda=880 \mathrm{~nm})$ passing through the dispersions. The apparatus has two synchronized detectors. A transmission detector 
recorded light passing through a probe under angle of $0^{\circ}$ in relation to the incident light direction. The second one is a backscattering detector registering the light scattered under angle of $135^{\circ}$. The obtained data are stored and converted by a computer program. The analyzed dispersion in a glass phial $(7 \mathrm{~cm}$ in length) was placed in a thermostated chamber. The changes in transmission and backscattering of light passing through the dispersions were monitored for $22 \mathrm{~h}$ (single scans were collected every 15 $\mathrm{min})$. The results are shown as intensities of transmission and backscattering (in \%) vs. time. These data used to calculate the Turbiscan stability index (TSI) (using TLab EXPERT 1.13 and Turbiscan Easy Soft):

$$
T S I=\sqrt{\frac{\sum_{i=1}^{n}\left(x_{i}-x_{B S}\right)^{2}}{n-1}}
$$

where $x_{i}$ is the average backscattering for each minute of measurement, $x_{B S}$ is the average $x_{i}, n$ is the number of scans (repetitions of single measurement during the total time of the experiment).

\section{Results and discussion}

\subsection{Adsorption of water-soluble polymers}

In general, the adsorption of polymers onto a solid surface from an aqueous solution is determined by interactions of polymer - solid surface, polymer - solvent, solvent - solid surface, and polymer - polymer. The polymer solvent interaction determines the conformation of the polymer in the solution and affects the conformation of the polymer in an adsorption layer.

Polymers studied can be adsorbed onto the silica surface by the hydrogen bonds between polar functionalities (such as $\mathrm{C}-\mathrm{OH}, \mathrm{C}-\mathrm{O}-\mathrm{C}$, $\mathrm{C}=\mathrm{O}$ ) of the polymer and surface silanols [1719]. The adsorption isotherms of PVA, PEO, and PVP can be assigned to the Langmuir type (Fig. 1). An increase in the molecular weight leads to an increase in the adsorption.

The adsorption and structure of the adsorption layer depend on the molecular weight of polymers $[1-3,20]$. The dependence of the adsorption on the molecular weight of polymers for sorbents composed of nonporous nanoparticles (NPNP) can be described by a simple equation [20].

$$
A_{\max }=\mathrm{K} \cdot M_{\mathrm{w}}{ }^{\alpha}
$$

where $A_{\max }$ is the maximal adsorption, $M_{\mathrm{w}}$ is the molecular weight, $\mathrm{K}$ and $\alpha$ are the constants dependent on the polymer nature, solvent type, and temperature. Equation (4) could be used to estimate conformation features of adsorbed polymer chains at a solid surface. If $\alpha$ is close to 0 the polymer is adsorbed onto a surface by a flat way, and the maximum adsorption is independent of the molecular weight of a polymer. If $\alpha$ is close to 1 the polymer is adsorbed by one tail and, accordingly, the maximum adsorption depends linearly on the molecular weight of a polymer. 
All intermediate cases can be considered as the adsorption of a polymer to form the loops and tails of various sizes which can be described through the value of $\alpha$. The values of the maximal adsorption of the polymers can be determined from the adsorption isotherms (Fig. 1). An increase in the adsorption with increasing molecular weight is observed for all the polymers. This suggests that the adsorption of PEO, PVA, and PVP proceeds with the formation of the loops and tails (Fig. 3, models [20]).

a)

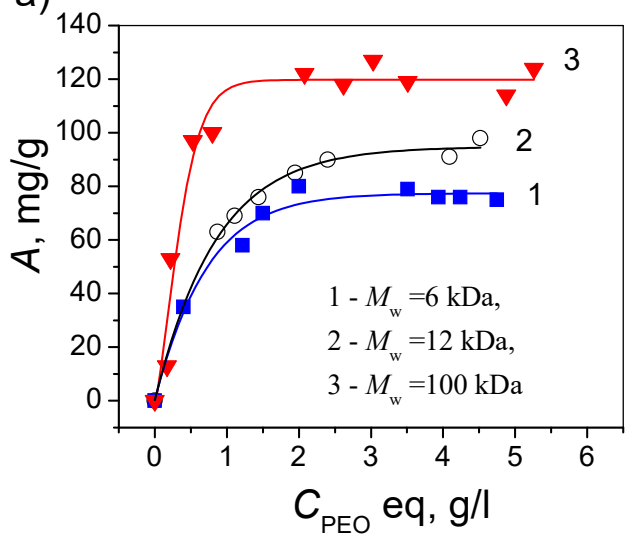

c)

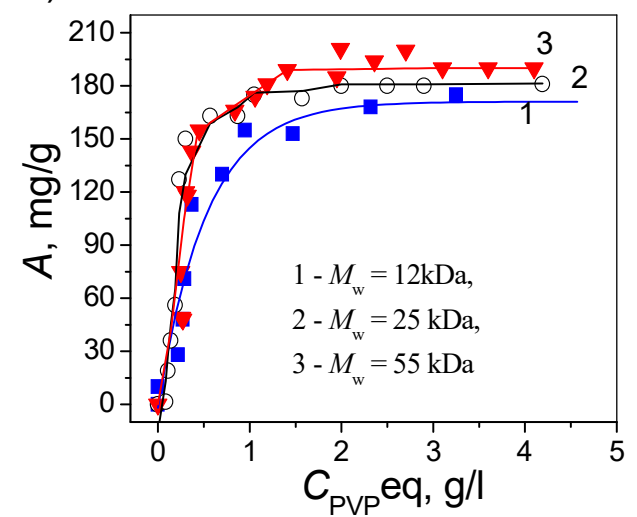

Changes in the Gibbs free energy (Fig. 1d) upon the polymer adsorption are relatively small due to several factors such as (i) great desolvation energy related to displaced water molecules bound to both a silica surface and macromolecule fragments contacted to the surface; (ii) strong polymer-polymer interactions (maximal for PVA among the polymers studied due to the hydrogen bonds between the $\mathrm{COH}$ groups) which change upon

Figure 1. The adsorption isotherms for: (a) PEO; (b) PVA; (c) PVP onto nanosilica A-300; (d) the distribution functions of the Gibbs free energy changes $(\Delta G)$ upon polymer adsorption onto a silica surface from the aqueous solutions for PEO (curve 1, $\mathrm{M}_{\mathrm{w}}=100 \mathrm{kDa}$ ), PVA (curve 2, $\mathrm{M}_{\mathrm{w}}=72 \mathrm{kDa}$ ), and PVP (curve $3, \mathrm{M}_{\mathrm{w}}=$ $55 \mathrm{kDa})$ 
the adsorption; (iii) conformational changes of the polymers; and (iv) changes in the particleparticle interactions. All these factors can reduce the changes in the Gibbs free energy upon the polymer adsorption. The length of the tails increases with increasing molecular weight of a polymer. This can be characterized by the coefficients in Eq. (2) calculated for each polymer (Table 1).

The determining factor for the adsorption is the number of functional groups capable to interact with the surface that is proportional to the chain length. Figure 2 shows the dependence of the number of the polymer chain units $(N)$ per a surface silanol on the total number of units in the polymer chain. Thereby, all the studied polymers are adsorbed with the formation of the loops and tails. PVA forms the longest tails, and PVP is most flatly sorbed.

Table 1. The maximal adsorption of polymers on silica surface and coefficients $K$ and $\alpha$ (equation 3 ) for polymers of various $M_{\mathrm{w}}$

\begin{tabular}{|c|c|c|c|c|c|c|c|c|c|}
\hline \multirow[b]{2}{*}{$M_{\mathrm{w}}, \mathrm{kDa}$} & \multicolumn{3}{|c|}{ PVA } & \multicolumn{3}{|c|}{ PVP } & \multicolumn{3}{|c|}{ PEO } \\
\hline & 43 & 60 & 72 & 12 & 25 & 55 & 6 & 12 & 100 \\
\hline$A_{\max }, \mathrm{mg} / \mathrm{g}$ & 120 & 25 & 32 & 171 & 181 & 191 & 80 & 91 & 124 \\
\hline$A_{\max }, \mathrm{mg} / \mathrm{m}^{2}$ & 0.50 & 0.52 & 0.55 & 0.71 & 0.75 & 0.80 & 0.32 & 0.38 & 0.52 \\
\hline$K$ & & 17.8 & & & 85.4 & & & 17.4 & \\
\hline$\alpha$ & & 0.18 & & & 0.07 & & & 0.17 & \\
\hline
\end{tabular}

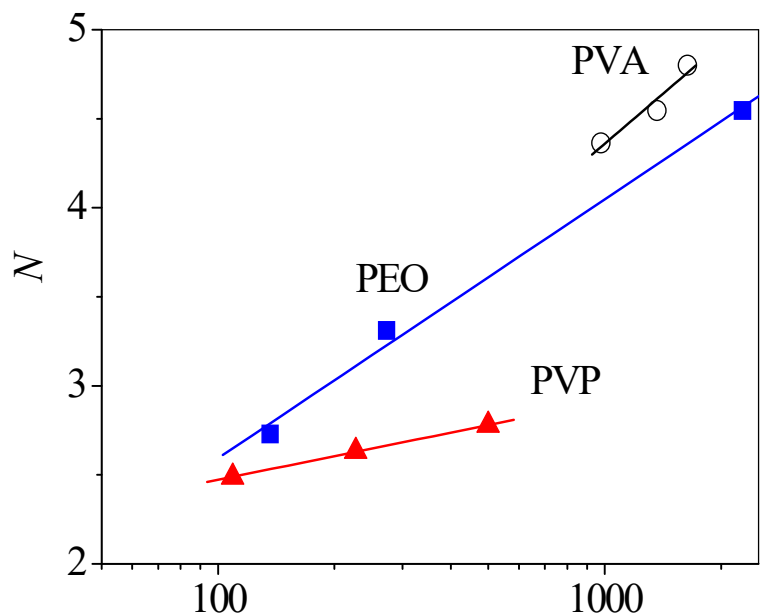

Total number of units in the polymer chain

Figure 2. Number of units of the polymer chain (N) corresponding to one surface silanol group vs. total number of units in the polymer chain

a



b

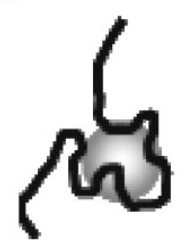

Figure 3. Models of interactions of a polymer chain with a particle: $\mathrm{a}$ - "long loops" model and b - "short loops" model [20]

\subsection{Rheological properties}

The adsorption of polymers changes the interparticle interactions and affects the formation of the dispersion structures. The viscosity of the dispersion depends on the viscosity of the dispersion medium, concentration of solid particles, particle sizes and their aggregation, and the interactions between the particles and macromolecules. The interparticle and intermolecular interactions determine the structure of dispersion and, therefore, its rheological properties. 
Figure 4 shows the rheological curves for the aqueous dispersion of nanosilica (5 wt. \%) prepared without ultrasonic treatment (Fig. 4, curve 1) and sonicated (Fig. 4, curve 2). The dependence of the effective viscosity of 5 wt. \% aqueous silica dispersion prepared without ultrasonic treatment on the shear rate (Fig. 4, curve 1) shows that it decreases with increasing shear rate. This reduction is typical for structured (non-Newtonian) liquids. It occurs due to the presence of the initial structure, which collapses under mechanical stress during the viscosity measurement. The processes, occurring during the measurements of the viscosity using a rotational viscometer, are characterized by a dynamic equilibrium between processes of destruction of interparticle bonds under the shear stress and their restoration or formation of new ones. At reverse reducing shear rate, the effective viscosity of the system increases due to partial reconstruction of destroyed interparticle bonds. Such rheological behavior, which is characterized by a decrease in the viscosity due to destruction of the bonds and a reverse increase in the viscosity due to recovery interparticle bonds, is thixotropic [22].

Typically, if the recovery rate of the interparticle bonds is less than the rate of their destruction that a hysteresis loop is observed in the rheograms. Its shape can be characteristic for the destruction and restoration of structures depending on the shear rate (Fig. 4, curve 1).

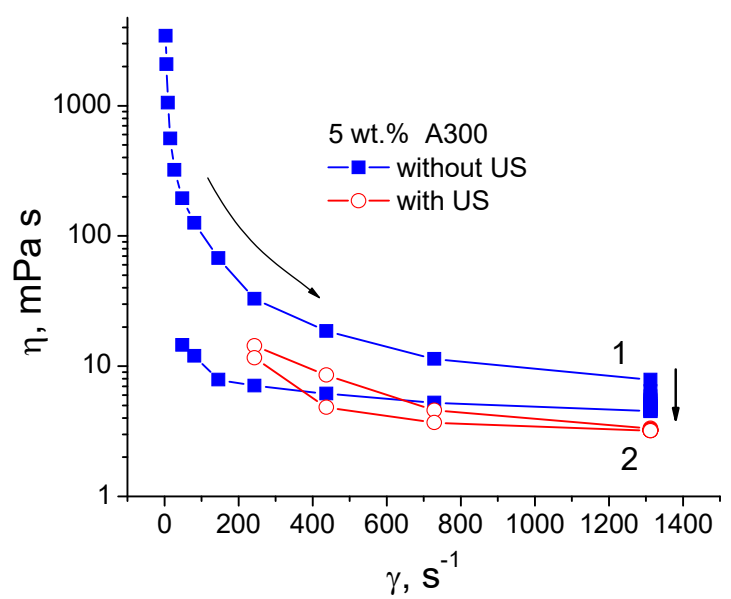

Figure 4. Dependences of viscosity $(\eta)$ on shear rate $(\gamma)$ for aqueous dispersions of silica prepared by stirring (curve 1) and by ultrasonic treatment (curve 2)

Ultrasonic treatment of the dispersion results both in the destruction of the interparticle bonds and a marked decrease in the viscosity (Fig. 4, curve 2). In $24 \mathrm{~h}$ after the ultrasonic treatment, the silica dispersion behaves as a freely dispersed system, i.e., the dependence of the effective viscosity on the shear rate is negligible, and there is no a decrease in the viscosity under a constant shear rate.

Addition of a polymer to the silica aqueous dispersion pre-sonicated leads to significant changes in the viscosity and rheological behavior. Addition of any amount of a polymer results in an increase in the effective viscosity due to the formation of certain super-structures. Figure 5 shows the dependence of the effective viscosity at the maximum shear rate $1312 \mathrm{~s}^{-1}$ (with maximum destroyed structures) on the polymer concentration. The dependences of the effective viscosity at $\gamma=1312 \mathrm{~s}^{-1}$ on the polymer content have extremes for all the 
systems. The maxima are observed at low polymer content. To determine features of this behavior, it is necessary to know the polymer amount adsorbed onto the silica surface and the polymer amount remained in the solution. From the comparison of the rheological data with the adsorption isotherms, one can see that initial increase in the viscosity is observed if the polymer is completely adsorbed. The amount of the polymer adsorbed onto the silica surface that corresponds to the maximum viscosity is 80 $\mathrm{mg} / \mathrm{g} \mathrm{SiO}_{2}$ (at initial polymer concentration $4 \mathrm{~g} / \mathrm{l})$, for PVA it corresponds to $0.5 \cdot A_{\max }$ and for PEO to $\left.0.7 \cdot A_{\max }\right)$ and $2.5 \mathrm{~g} / 1$ (PVP at $\left.0.3 \cdot A_{\max }\right)$. An increase in the viscosity of polymer/silica/water can be explained by the formation of polymer bridges between silica nanoparticles from neighboring NPNP aggregates (corresponding to the first state upon the structure formation toward the maximal viscosity) [14]. If the silica surface is completely covered by the polymer the number of interparticle (inter-aggregate) bridges strongly decreases and the system remains (or becomes through re-dispersion) freely dispersed.

This second condition results in a decrease in the effective viscosity with increasing polymer concentration. The polymer chains are incompletely bound to the silica surface, and a significant part of them remains in the solution. a)

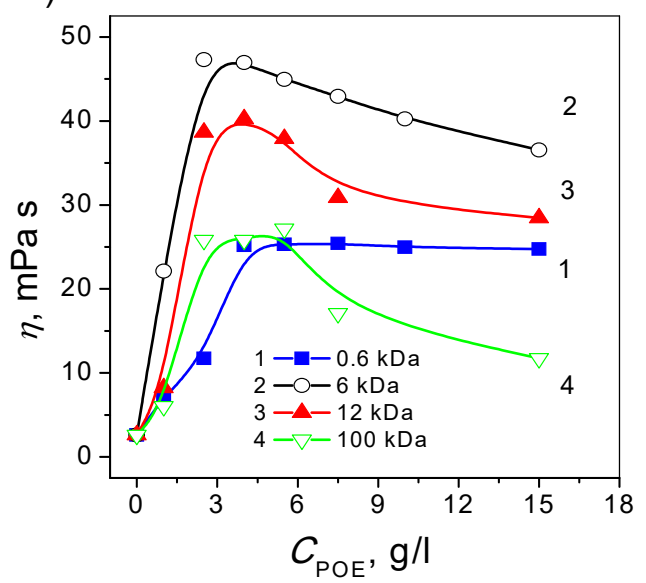

b)

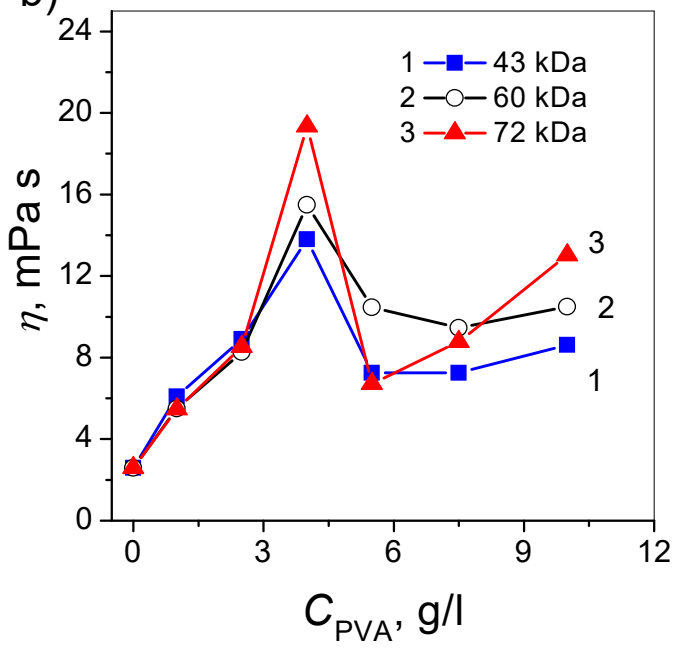

c)



Figure 5. Dependence of viscosity of aqueous dispersions of silica (5 wt. \%) as a function of polymer concentration at the constant shear rate $1312 \mathrm{~s}^{-1}$ for systems containing PEO (a), PVA (b), PVP (c) 
The third state, when the maximum adsorption is reached and the excess polymer remains in the solution in the unbound state can be characterized by an increase in the viscosity of whole system due to increasing viscosity of the dispersion medium with increasing polymer concentration. Thus, the viscosity of the silica dispersions increases with increasing concentrations of PVA having a sufficiently large molecular weight (as well strong polymerpolymer interactions) and viscosity of the aqueous solution (Fig. 5b, curve 2). For the silica dispersion in the presence of PEO and PVP $(\mathrm{Mw}=55 \mathrm{kDa}$ and $12 \mathrm{kDa}$, respectively) such increase in the viscosity is not observed (Fig. 5a,c, curves 1 and 3). This is possible due to the differences in the behavior of these polymers in the solution and during the formation of the adsorption layers, and the absence of the hydrogen bonding in PEO and PVP macromolecules per se in contrast to PVA.

The values of the aforementioned effective viscosity correspond to the equilibrium viscosity, when the rates of destruction and restoration of the interparticle bonds are equal. According to [22], the reduction of the viscosity at a constant shear rate corresponds to the thixotropic behavior of the system when the equilibrium shifts under mechanical stress toward the destruction of the interparticle bonds. The increase in the viscosity at a constant shear rate corresponds to the rheopectic behavior of the system when the equilibrium shifts under mechanical stress toward the formation of new interparticle bonds. The rheopectic behavior is less common than the thixotropic one and it depends strongly on the characteristics of the interparticle interactions. The aqueous dispersion of silica is characterized by the thixotropic behavior (Fig. 4, curve 1 and [23]), but in the presence of the polymers the rheological properties can change toward the rheopectic ones.

The shear rate dependences of the viscosity and 3D-shear rate dependences on the viscosity and time for the dispersions of silica in the presence of PVA, PVP, and PEO are shown in Figures 6 and 7. The dispersions of silica in the presence of PVA demonstrate a typical thixotropic behavior. The destruction of the dispersion structure occurs with increasing shear rate (Fig. 6a). Monitoring of the system at a constant shear rate vs. time and then at decreasing shear rate shows partial restoration of the structure. This rheological behavior is observed at various concentrations and molecular weights of PVA.

The rheological behavior of the silica dispersion in the presence of PVP or PEO depends on the polymer concentration. For the dispersion at $4 \mathrm{~g} / \mathrm{l}$ of PVP, there is a considerable increase in the effective viscosity upon exposing system at a constant shear rate. The viscosity values decreasing significantly at a reverse shear rate exceed the initial ones (Fig. 7a). 
a)
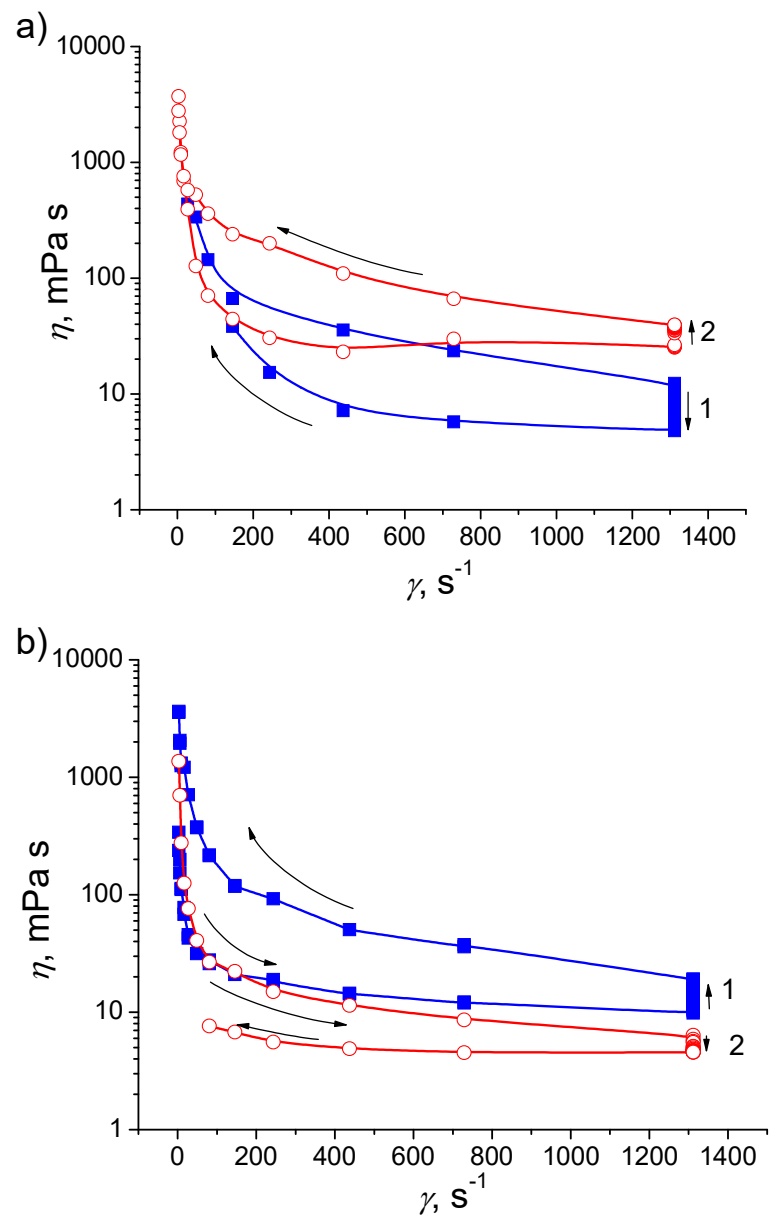

Figure 6. Viscosity $(\eta)$ vs shear rate $(\gamma)$ for aqueous dispersions of $\mathrm{SiO}_{2}$ in presence of PVA $\left(M_{\mathrm{w}}=43 \mathrm{kDa}\right.$, $\left.C_{\mathrm{PVA}}=4 \mathrm{~g} / \mathrm{l}\right)(\mathrm{a}-$ curve 1$), \mathrm{PEO}\left(100 \mathrm{kDa}, C_{\mathrm{PEO}}=4 \mathrm{~g} / \mathrm{l}\right)(\mathrm{a}$ - curve 2) and PVP $55 \mathrm{kDa}$ (b) with $C_{\mathrm{PVP}}=4 \mathrm{~g} / \mathrm{l}(\mathrm{b}-$ curve 1) and $\mathrm{C}_{\mathrm{PVP}}=15 \mathrm{~g} / \mathrm{l}(\mathrm{b}-$ curve 2$)$

This system shows explicit rheopectic properties that can be attributed to the formation of additional bridging bonds between the silica particles due to the mechanical loading onto the dispersion. The same rheopectic behavior is typical for the aqueous silica dispersions with PEO (Fig. 6b).

The dispersions of silica in the presence of PVA demonstrate a typical thixotropic behavior. The destruction of the dispersion structure occurs with increasing shear rate (Fig. 6a).
Monitoring of the system at a constant shear rate vs. time and then at decreasing shear rate shows partial restoration of the structure. This rheological behavior is observed at various concentrations and molecular weights of PVA.
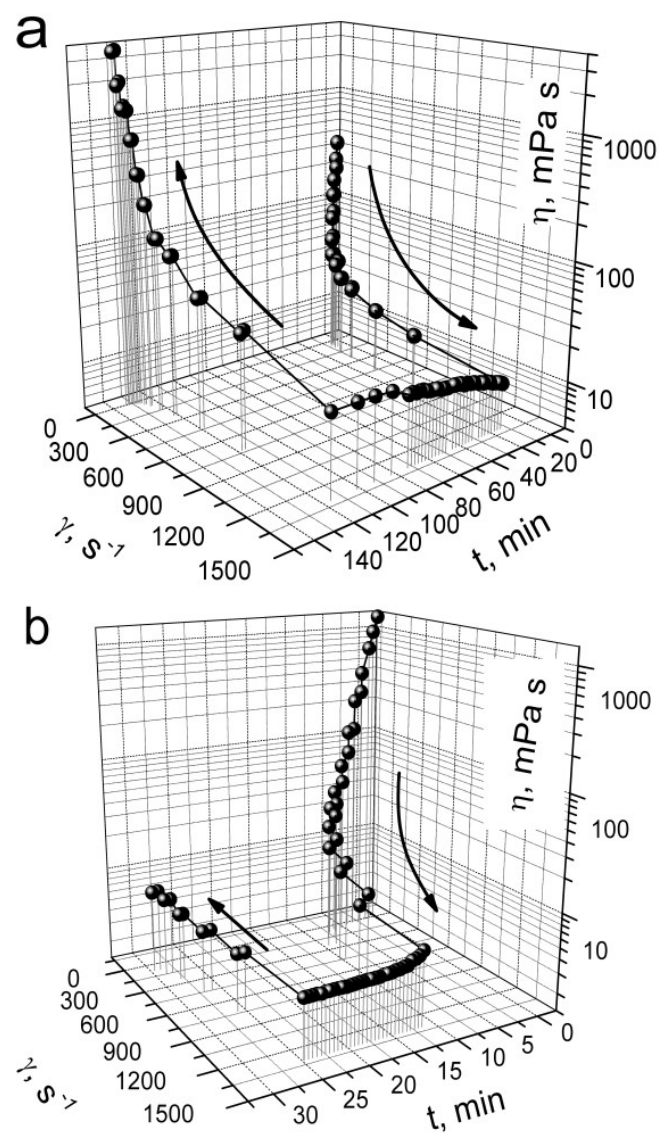

Figure 7. Three-dimensional rheological curves for aqueous dispersions of silica prepared by ultrasonic treatment in presence of PVP $55 \mathrm{kDa}$ with $\mathrm{C}_{\mathrm{PVP}}=4 \mathrm{~g} / 1$

$$
\mathrm{SiO}_{2}(\mathrm{a}) \text { and with } \mathrm{C}_{\mathrm{PVP}}=15 \mathrm{~g} / \mathrm{l}(\mathrm{b})
$$

The rheological behavior of the silica dispersion in the presence of PVP or PEO depends on the polymer concentration. For the dispersion at $4 \mathrm{~g} / 1$ of PVP, there is a considerable increase in the effective viscosity upon exposing system at a constant shear rate. The viscosity values decreasing significantly at 
a reverse shear rate exceed the initial ones (Fig. 7a). This system shows explicit rheopectic properties that can be attributed to the formation of additional bridging bonds between the silica particles due to the mechanical loading onto the dispersion. The same rheopectic behavior is typical for the aqueous silica dispersions with PEO (Fig. 6b). With increasing PVP concentration to $15 \mathrm{~g} / \mathrm{l}$, the system becomes again thixotropic, since the entire silica surface is covered by the polymer. Thus, there is no place for the formation of new bridging bonds (Fig. 7b). For the silica dispersions with PEO, the concentration dependence of the viscosity is also observed. However, these systems are characterized by the rheopectic behavior at high concentration of the polymer (greater than monolayer coverage), which indicates the differences in the structure formation. Perhaps, this behavior is due to the differences in the interactions between the silica nanoparticles covered by PVP and PEO having different polar functionalities. The silica dispersions with PEO are characterized by the highest viscosity and manifestation of rheopexy almost for all studied polymer concentrations. The rheopectic behavior may be associated with the flow with the orientation mechanism, which is a common characteristic of the polymer solutions, and the formation of additional bridging bonds between nanoparticles under dynamic conditions.

Thus, on the basis of the rheological studies, the mechanism of the structure formation in the aqueous dispersions of fumed silica in the presence of non-ionic polar polymers can be analyzed in detail (Fig. 8). At low concentrations of a polymer, long linear macromolecules form bridges between silica nanoparticles which could belong to neighboring aggregates of NPNP. Each macromolecule can interact with several nanoparticles that contribute to a sharp increase in the viscosity.


Figure 8. Scheme of interactions between the polymer macromolecules and oxide particles in aqueous dispersions with various polymer contents

This effect can depend on the nature of a polymer (structure and conformation of macromolecules in the solution, molecular weight, the nature of polymer functionalities, etc.). Maximal bridge-formation (corresponding to the maximal viscosity) is observed at initial polymer concentrations providing $50-70 \%$ of the monolayer polymer adsorption. Further increase in the polymer concentration leads to the formation of a thicker adsorption layer at the silica particles (aggregates). This prevents the formation of polymer bridges between neighboring aggregates or individual NPNP. Therefore, the viscosity decreases. These processes of the structure formation determine 
the rheological parameters of the dispersions, their aggregative and sedimentation stability.

\subsection{Stability of dispersions}

The turbidimetric data for the silica dispersions at concentration of 5 and 1 wt. \% show that the values of the transmission are quite large, more than $60 \%$ that indicates the small size of silica particles (NPNP aggregates). However, the TSI index (Table 2) testifies that the particle size changes with time, i.e. the aggregation increases. However, the sedimentation separation is not observed (Table 2).The aggregative and sedimentation stability corresponds to the most stable systems at TSI close to 0 , and at TSI close to 100 , the systems are minimum stable. Thus, 5 and 1 wt. \% silica dispersions are relatively stable systems. To improve the stability or flocculation, water-soluble polymers can be added to the nanosilica dispersions.

Table 2. Dependence of the stability index TSI on time after preparation of dispersion for initial fumed silica without polymer with concentrations of solid $5 \mathrm{wt} . \%$ and 1 wt. $\%$

\begin{tabular}{|c|c|c|c|c|c|c|}
\hline$t, \mathrm{~h}$ & 0.17 & 1 & 5 & 10 & 12 & 22 \\
\hline $\begin{array}{c}\text { TSI for 1 \% } \\
\mathrm{SiO}_{2}\end{array}$ & 0.2 & 1.5 & 4.5 & 6.4 & 7.0 & 8.0 \\
\hline $\begin{array}{c}\text { TSI for 5 \% } \\
\mathrm{SiO}_{2}\end{array}$ & 0.5 & 2.3 & 4.3 & 5.3 & 5.7 & 6.5 \\
\hline
\end{tabular}

As stated above, depending on the nature, concentration, length of the polymer chains, the polymers can act as stabilizers or flocculants. Based on the above rheological studies, the polymers are capable to form the bridges between the silica particles at a low concentration of the dispersion, when the silica surface is incompletely covered by the polymer. Therefore, to stabilize the dispersion, the polymer concentration should be equal or greater than that providing the adsorption monolayer. If polymers have only one type of the active functionalities (e.g. the $\mathrm{C}=\mathrm{O}$ groups in PVP ( $\mathrm{N}$ atoms in the PVP side rings are rather passive in the adsorption) and $\mathrm{C}-\mathrm{O}-\mathrm{C}$ in PEO), the polymer-polymer interactions are much weaker than the interactions of polymersilica surface and polymer-water caused by the formation of strong hydrogen bonds $\mathrm{SiO}-\mathrm{H} \cdots \mathrm{O}=\mathrm{C}<$ or $\mathrm{SiO}-\mathrm{H} \cdots \mathrm{O}\left(\mathrm{CH}_{2}\right)_{2}<$. Another case is for PVA because it has $\mathrm{C}-\mathrm{O}-\mathrm{H}$, which can form strong hydrogen bonds for both polymer-polymer and polymer-silica surface or polymer-water molecules. Therefore, there is another bridge mechanism for stabilizing dispersions, when polymers and dispersed particles form a continuous structure that is sedimentation stable due to loss of the mobility.

For colloidal stability investigation, the silica concentrations were chosen as 1,3 , and 5 wt. $\%$ and the polymer concentrations were selected (based on the adsorption data) close to and above that providing the monolayer adsorption.

Table 3 shows the TSI for 5 wt. \% silica dispersions with PEO of various $M_{\mathrm{w}}$ at initial concentrations of 4 and $6.5 \mathrm{~g} / \mathrm{l}$. The PEO 
adsorption onto the silica surface at the abovementioned concentrations more strongly enhances the sedimentation stability (aggregative stability) of the concentrated silica dispersions. The stability of 1 wt. $\%$ dispersions is lower than that at 5 wt. \% (Table 3).

Table 3. TSI for 5 wt. $\%$ and 1 wt. $\%$ silica aqueous dispersions in the presence of PEO $\left(M_{\mathrm{w}}=6 \mathrm{kDa}\right)$

\begin{tabular}{|c|c|c|c|c|c|c|}
\hline & \multirow{2}{*}{\begin{tabular}{c}
$\mathrm{C}_{\text {PEO, }}$ \\
$\mathrm{mg} / \mathrm{g}$ \\
\cline { 3 - 7 }
\end{tabular}} & \multicolumn{5}{|c|}{$t, \mathrm{~h}$} \\
\cline { 3 - 7 } & 0.17 & 1 & 10 & 15 & 18 \\
\hline $\begin{array}{c}\mathrm{TSI} \\
\text { for } \\
5 \%\end{array}$ & 25 & 0.4 & 0.7 & 0.8 & 0.9 & 1 \\
\cline { 2 - 7 } & 80 & 0 & 0 & 0 & 0 & 0.1 \\
\cline { 2 - 7 } $\mathrm{SiO}_{2}$ & 130 & 0 & 0.2 & 0.26 & 0.28 & 0.3 \\
\hline $\begin{array}{c}\mathrm{TSI}_{2} \\
\text { for } \\
1 \%\end{array}$ & 25 & 1.4 & 4.1 & 8.4 & 9.3 & 9.9 \\
\cline { 2 - 7 } & 80 & 0.1 & 0.8 & 2.2 & 2.8 & 3.0 \\
\cline { 2 - 7 } $\mathrm{SiO}_{2}$ & 130 & 0.2 & 1.3 & 2.3 & 2.7 & 2.9 \\
\hline
\end{tabular}

The influence of the polymer concentration on the stability of the aqueous dispersions of silica is a non-monotonic function. Significantly lower stability for 5 and 1 wt. $\%$ silica dispersions is observed at a low polymer content $(1.25 \mathrm{~g} / \mathrm{l})$. At higher concentrations of PEO (4 and $6.5 \mathrm{~g} / 1)$, the dispersions are characterized by a higher stability. This pattern is due to the fact that the concentration of PEO $(6 \mathrm{kDa})$ is less than the adsorption monolayer (Fig. 1a), but at higher concentrations of the silica, it does not lead to the loss of the dispersion stability in contrast to the low-concentrated dispersions. Thus, the stability of the aqueous dispersions of silica in the presence of PEO depends strongly on the silica and polymer concentrations and slightly on the polymer molecular weight. Moreover, the TSI stability factor for the stable dispersions is virtually unchanged with time, while for the unstable dispersions; its growth reflects the destabilization kinetics.

For the silica dispersions in the presence of PVP, there are significant differences in the stability of the dispersions at initial PVP concentrations less than $50 \%$ of monolayer $(4 \mathrm{~g} / \mathrm{l})$ and at $8.75 \mathrm{~g} / 1$ (greater than the monolayer). For the second case, it is possible to observe the improvement of the dispersion stability compared to the dispersion of silica alone. At low PVP concentration, there is a significant decrease in the stability of the dispersions (Fig. 9). The dependence on the silica concentration is similar to that for the silica dispersions in the presence of PEO. For 5 wt. \% dispersions, there is a higher stability than at 1 wt. $\%$ in the presence of any polymer studied.

For the silica dispersions with PVA, there are significant differences in the stability depending on the concentration of PVA (Fig. 10a). The monolayer PVA is about 120$130 \mathrm{mg} / \mathrm{g} \mathrm{SiO}_{2}$, but the impact of the bridge formation on the stability at PVA concentration below the monolayer formation $(4 \mathrm{~g} / \mathrm{l})$ is observed only for the dispersions at a low concentration of the silica. 
a)

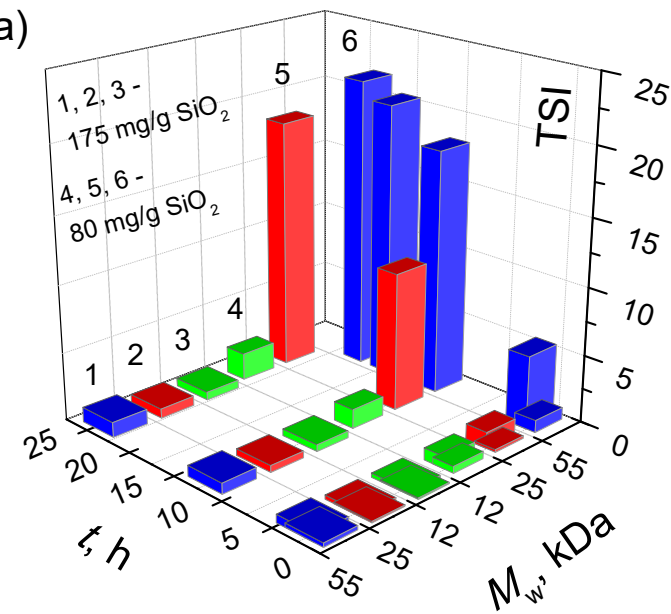

b)

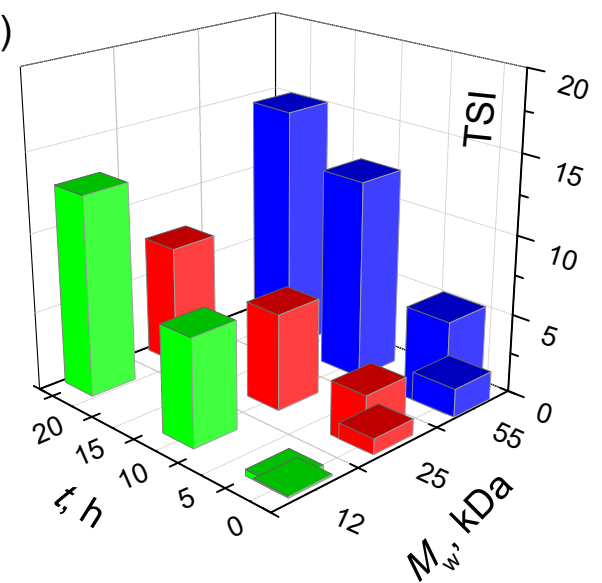

Figure 9. Stability index TSI for 5 wt. \% (a) and 1 wt. \% (b) aqueous dispersions of silica in the presence of PVP with different $M_{\mathrm{w}}\left(\mathrm{C}_{\mathrm{PVP}}=4 \mathrm{~g} / 1\right.$ and $0.8 \mathrm{~g} / 1$ corresponds to $80 \mathrm{mg} / \mathrm{g} \mathrm{SiO}_{2}$ )

The aqueous dispersion of silica with PVA is stable and its stability increases with increasing molecular weight of the polymer (Fig. 10b) or with increasing concentration of the silica (Fig. 10c). Even for dispersions with low aggregative stability, the sedimentation separation is not observed. a)
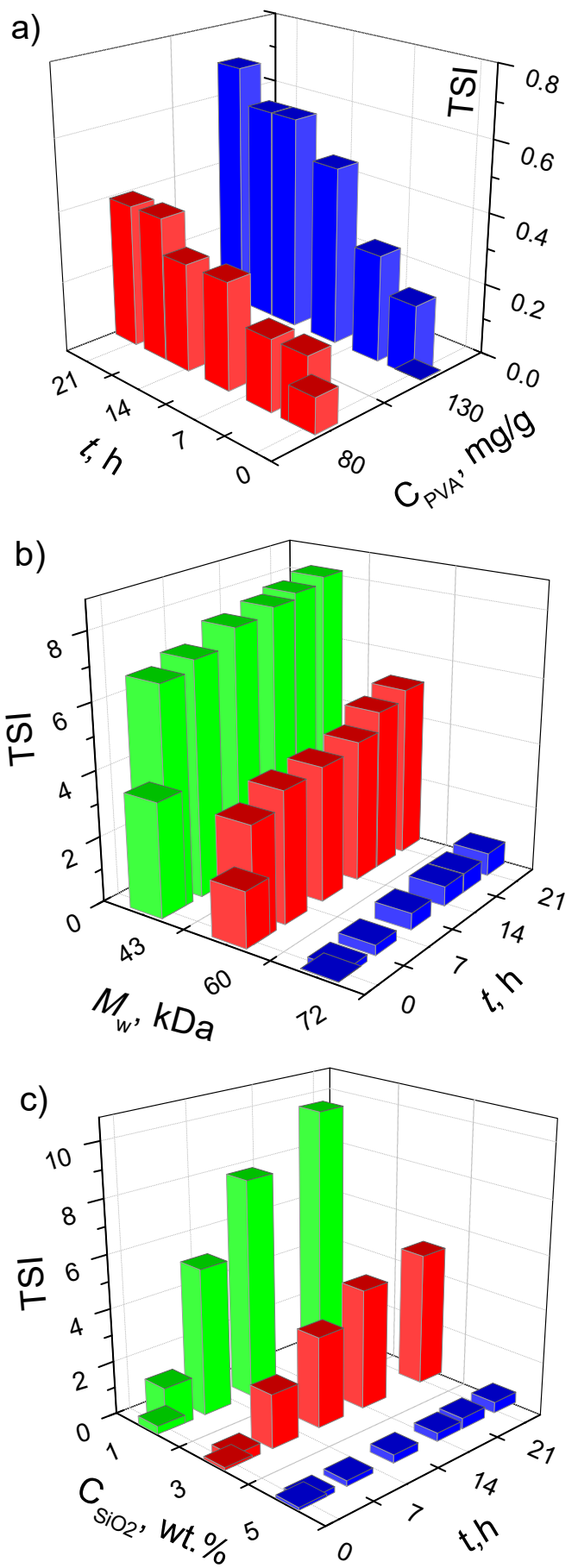

Figure 10. TSI for aqueous dispersions of silica in the presence of PVA: (a) with different $C_{\mathrm{SiO}_{2}}$ and $C_{\mathrm{PVA}}=4 \mathrm{~g} / 1$ $\mathrm{SiO}_{2}\left(M_{\mathrm{w}}=72 \mathrm{kDa}\right)$, (b) $C_{\mathrm{SiO}_{2}}=5$ wt. $\%$ and $C_{\mathrm{PVA}}=6.5 \mathrm{~g} / 1$ $\mathrm{SiO}_{2}$ with different $M_{\mathrm{w}}$ and (c) with concentration of $C_{\mathrm{SiO}_{2}}$ $=5$ wt. $\%$ in the presence of different PVA $\left(M_{\mathrm{w}}=72 \mathrm{kDa}\right)$ concentration 


\section{Conclusions}

The adsorption of water-soluble polymers PEO, PVA, and PVP of various molecular weights on the nanosilica surface from the aqueous solutions and their effects on the aggregative and sedimentation stability and rheological properties of the dispersions were studied. The maximal adsorption of the polymers increases with increasing their molecular weight. This indicates that PEO, PVA, and PVP adsorption proceeds with the formation of loops, trains, and free tails. The relative contribution of trains decreases with increasing molecular weights. Poly(vinyl alcohol) molecules form the longest tails and the poly(vinyl pyrrolidone) is sorbed most flatly due to features of the functionalities: $\mathrm{COH}$ in PVA (forming the strong hydrogen bonds for polymer-polymer, polymer-silica surface, polymer-water molecules) and $\mathrm{C}=\mathrm{O}$ in $\mathrm{PVP}$ (no hydrogen bonds in polymer-polymer), respectively. The structure of the adsorption layer determines the interactions between the particles in aqueous dispersions and, thus, such parameters as the dispersion stability and rheological properties. The study of the rheological properties of the aqueous dispersions of nanosilica in the presence of PEO, PVA, and PVP has showed that the dispersions are characterized by extreme dependence of the viscosity on the polymer concentration. The highest values of viscosity are observed at the initial polymer concentration of $50-70 \%$ of monolayer. This is due to the formation of the polymeric bridges between the silica nanoparticles. With an increase in the polymer concentration, a denser adsorption layers around the particles is formed which prevents the formation of polymeric bridges between them, and the viscosity decreases. The sedimentation stability of the aqueous silica dispersions in the presence of the polymers depends on the silica and polymer concentrations and the molecular weight of the polymers. The dispersion stability increases with increasing concentration of the solid phase in the presence of all polymers studied, but the increase in the stability with increasing molecular weight is characteristic only for the dispersion in the presence of PVA. The comparison of the rheological data with the sedimentation stability of the dispersions shows that it is possible to observe two mechanisms of the stabilization. The first one is due to the formation of dense adsorption polymer layers around the silica nanoparticles that prevents the formation of polymeric bridges between neighboring silica nanoparticles. The second one is due to the formation of the continue clusters in the dispersion system due to strong polymer-polymer (e.g. PVA) interactions (similar to the solution of the polymer alone) that prevent the sedimentation processes.

\section{References}

[1] Fleer G. et al. Polymers at Interfaces, Chapman and Hall, 1993. 
[2] Lyklema J. Fundamentals of Interface and Colloid Sciences, vol. II, Academic Press, 1995

[3] J. Gregory, S. Bárány: Adsorption and flocculation by polymers and polymer mixtures. Adv.Colloid Interf.Sci., 168 (2011) 1-12. (Q1 in "Colloid a. Surface Chemistry")

[4] Napper DH. Polymeric Stabilization of Colloidal Dispersions. London: Academic Press, 1983.

[5] Gu S, Shi Y, Wang L, Liu W, Song Z. Study on the stability of modified colloidal silica with polymer in aqueous environment. Colloid Polym Sci 2014;292:267273. DOI 10.1007/s00396-013-3109-4.

[6] Dobias B, Stechemesser H (Ed). Coagulation and Flocculation. 2nd Ed. CRC Press.2005. DOI: 10.1201/9781420027686. ch11

[7] Jeon SJ, Hong WH. Effects of Poly(acrylic acid) and Poly(ethylene oxide) Adsorption on the Stability of Alumina Suspension. Korean J Chem Eng 2003; 20(5): 916-921

[8] Bhatti QA, Baloch MK, Schwarz S, Petzold G. Effect of Various Parameters on the Stability of Silica Dispersions. J Solution Chem 2014; 43:1916-1928. DOI 10.1007/s10953-014-0260-6

[9] Bauer D, Killmann E, Jaeger W. Flocculation and stabilization of colloidal silica by the adsorption of polydiallyl-dimethyl-ammoniumchloride (PDADMAC) and of copolymers of DADMAC with N-methyl-Nvinylacetamide (NMVA). Colloid Polym Sci 1998; 276: 698-708

[10] Mpofu P, Addai-Mensah J, Ralston J. Investigation of the effect of polymer structure type on flocculation, rheology and dewatering behavior of kaolinite dispersions. Int J Miner Process 2003:71(1-4): 247-268.

[11] Wiśniewska M, Terpiłowski K, Chibowski S, Urban T, Zarko VI, Gun'ko VM.Effect of solution $\mathrm{pH}$ on the stability of mixed silica-alumina suspension in the presence of polyacrylic acid (PAA) with different molecular weights. Cent Eur J Chem 2013; 11:101-110. DOI: $10.2478 / \mathrm{s} 11532-012-0136-9$.
[12] Saito Y, Ogura H, Otsubo Y. Rheological behavior of silica suspensions in aqueous solutions of associating polymer. Colloid Polym Sci 2008; 286:15371544. DOI 10.1007/s00396-008-1928-5

[13] Saito Y, Hirose Y, Otsubo Y. Effect of poly(ethylene oxide) on the rheological behavior of silica suspensions. Rheol Acta, 2011;50:291-301. DOI 10.1007/s00397-010-0523-0

[14] Ilyin SO, Kulichikhin VG, Malkin AYa. Rheological properties of emulsions formed by polymer solutions and modified by nanoparticles. Colloid Polym Sci, 2015; 293(6): 1647-1654. DOI 10.1007/s00396-0153543-6

[15] Gun'ko VM, Turov VV. Nuclear Magnetic Resonance Studies of Interfacial Phenomena, CRC Press, Boca Raton, 2013

[16] Provencher SW. A constrained regularization method for inverting data represented by linear algebraic or integral equations. Comp Phys Comm 1982; 27: 213227.

[17] Gun'ko VM, Pissis P, Spanoudaki A, Zarko VI, Nychiporuk YM, Andriyko LS, Goncharuk EV, Leboda R, Skubiszewska-Zięba J, Osovskii VD, Ptushinskii YG. Relaxation phenomena in poly(vinyl alcohol)/fumed silica affected by interfacial water. Journal of Colloid and Interface Science 2007; 312(2): 201-213. DOI:10.1016/j.jcis.2007.03.065

[18] Gun'ko VM, Voronin EF, Zarko VI, Goncharuk EV, Turov VV, Pakhovchishin SV, Pakhlov EM, Guzenko NV, Leboda R, Skubiszewska-Zieba J, JanuszW, Chibowski S, Chibowski E, Chuiko AA. Interaction of poly(vinyl pyrrolidone) with fumed silica in dry and wet powders and aqueous suspensions. Colloids and Surfaces A: Physicochemical and Engineering Aspects 2004; 233(1-3): 63-78. DOI:10.1016/j.colsurfa.2003.11.024

[19] Voronin EF, Gun'ko VM, Guzenko NV, Pakhlov EM, Nosach LV, Leboda R, Skubiszewska-Zieba J, Malysheva ML, Borysenko MV, Chuiko AA. Interaction of poly(ethylene oxide) with fumed silica. J Colloid 
Interface

Sci.

2004;279(2):326-40.

DOI:10.1016/j.jcis.2004.06.073

[20] Mizuhara K, Hara K, Imoto T. Adsorption of polymers at the solution-solid interfaces. KolloidZeitschrift und ZeitschriftfürPolymere1970;238: 442-446. DOI: $10.1007 / \mathrm{BF} 02085570$

[21] Pomogailo AD, Rosenberg AS, Uflyand IE Metal nanoparticles in polymers. Moscow:Chemistry, 2000.

[22] Tadros T. Rheology of Dispersions: Principles and Applications. Wiley-VCH, 2010, DOI:10.1002/9783527631568

[23] Barnes HA. Thixotropy - a review. J NonNewtonian Fluid Mech1997;70:1-33. DOI: 10.1016/S0377-0257(97)00004-9 